\title{
Dengue seropositivity in a randomly selected sample from Yucatan analyzed in the context of dengue cases reported between 1996 and 2006
}

\author{
S. Gómez-Carro ${ }^{1}$, N. Méndez-Domínguez ${ }^{* 2}$, J. F. Mendez-Galván ${ }^{3}$ \\ ${ }^{1}$ Hospital General "Dr. Agustín O'Horán", Mérida, México \\ ${ }^{2}$ Centro de Investigación y de Estudios Avanzados del IPN, Departamento de Ecología Humana, Mérida, México \\ ${ }^{3}$ Hospital Infantil de México Federico Gómez, México City, México
}

Received: August 10, 2016

DOI: $10.5430 /$ jer.v3n1p23
Accepted: September 18, 2016 Online Published: September 19, 2016

URL: http://dx.doi.org/10.5430/jer.v3n1p23

\begin{abstract}
Introduction: Dengue virus (Denv) was first reported in Yucatan in 1979. Since then, Denv has been associated with multiple cases of Dengue Fever (DF) and Dengue Hemorrhagic Fever (DHF), becoming an endemic disease in Yucatan, Mexico.

Objectives: To determine the seropositivity to dengue infections in random sample of the Yucatan general population in 1996 and 2006 and analyze it along with the reported dengue cases in the state during that ten-year-period.

Methods: Samples from the randomly selected participants were tested for IgG dengue antibodies in both serosurveys, while laboratory confirmed DF and DHF were obtained from the epidemiologic surveillance system from 1996 to 2006.

Results: The overall seropositivity to Denv infection was $59.9 \%$ in 1996 and $81.5 \%$ in 2006, according to the serosurveys. The increase in seropositivity can be at least partially explained by the peak in DF and DHF cases that took place in 1997, as reported in the surveillance system.

Conclussion: Data drawn from the 1996 and 2006 serosurveys showed an increase of seropositivity to Denv infections, which can partially be explained by the 1997 outbreak in the Yucatan. While seroprevalence studies were useful to identify the proportion of seropositive population, the case reports from the epidemiologic surveillance system were useful to identify the epidemic year, meaning that both sources of information are complementary to better understand the Denv dynamics during the ten-year-period elapsed between 1996 and 2006.
\end{abstract}

Key Words: Dengue, Seroprevalence, Yucatán, Elisa, Immunoglobulin G

\section{INTRODUCTION}

Dengue virus (Denv) preventive strategies for eradicating Aedes aegipty were adopted by Mexican government in the 1960's decade, and as a result, Aedes borne diseases were not reported for over a decade. By the end of the 1970's, in Mexico, the first confirmed dengue cases were reported in Chiapas. Dengue Fever (DF) cases were confirmed in Yucatan in 1979, rapidly followed by major epidemics in the State for the next five years, and with the appearance of dengue serotype 4 in Yucatan during 1984, Dengue Hemorrhagic Fever (DHF) incidence increased dramatically. The serosurveys conducted during the 1980's decade showed a

*Correspondence: N. Méndez-Domínguez; Email: ninuxka@hotmail.com; Address: Centro de Investigación y de Estudios Avanzados el IPN Unidad Mérida, Departamento de Ecología Humana, Unidad Mérida Km, 6 Antigua carretera a Progreso Apdo, Cordemex 97310, Mérida, Yucatán, México. 
positivity close to $70 \%$ of the urban population, suggested a possible underreport of dengue cases at that time in $\mathrm{Yu}$ catan. ${ }^{[1-3]}$

Since then, Denv has been associated in Yucatan with multiple fatal cases, Dengue Fever DF and DHF, becoming an endemic disease which had been favored by the abundance of Aedes aegypti, the main dengue vector in the region. ${ }^{[4,5]}$

In Mexico, and particularly in Yucatan, experts have considered that the number of unreported DF cases were ten times more than reported by the epidemiological surveillance system. The prevalence of antibodies due to historic dengue infections could help to understand the Denv transmission if we consider the possibility that cases diagnosed and reported are not reflecting accurately the number of people that have been infected with at least one serotype of Denv. ${ }^{[6]}$

The recent Denv epidemic outbreak experienced during 2015 in Yucatan (with 17,016/1,502 Suspected/Confirmed Dengue Reported Cases) motivated us to look back and analyze the Denv dynamics over a ten-year period, including data drawn from both, passive surveillance and two seroprevalence studies. $^{[5]}$

Our aim in this study is to determine the seropositivity to dengue infections in a random sample of the Yucatan general population in 1996 and 2006 and to analyze that information considering the number of dengue confirmed cases reported by the epidemiologic surveillance system during that tenyear-period.

\section{MethodS}

\subsection{Seroprevalence surveys}

We chose the ten-year-period elapsed between 1996 and 2006 because the main author supervised and conducted serosurveys in those years, but also considering the access and availability of Denv reported cases in Yucatan during that period. The studies were undertaken in Yucatan, Mexico. First, in 1996 the participating communities were randomly chosen from a list of the 106 municipalities, including all communities with $\geq 15,000$ inhabitants, according to the official demographic records from the National Council of Population (CONAPO in Spanish) for 1996. The six selected municipalities were: Progreso, Uman, Chemax, Hunucma, Tizimin and Merida. As municipalities include smaller population units called communities, for the 2006 serosurvey, the list of communities from the municipalities selected for the 1996 serosurvey was randomized, that means that the participating individuals in 1996 and 2006 belonged to the same municipalities that were selected for the 1996 serosurvey, but were not necessarily from the same community. The population from the randomized municipalities together account for $75.3 \%$ of the total state population $(1,830,893$ inhabitants, according to CONAPO estimates for 2006).

Participants were randomly selected without replacement based on the geographic location of their homes through a geographical mapping system, by listing, numbering and then randomizing the row number of the districts and/or quadrants of the selected communities in an excel dataset. Only one participant was selected per household, and that participant was also selected randomly from among the inhabitants of the household, as every member of the family was coded with a number and chosen randomly in situ. Participants were visited at their homes simultaneously during the low dengue transmission season (dry season from January to April).

These district, quadrant, house and family member selection procedures were followed in both, the 1996 and the 2006 serosurveys, as both surveys were conducted and supervised mainly by the same research group and principal investigator. All participants signed an informed consent form voluntarily (in the case of minors, legal tutors signed consent forms). An inclusion criterion was that participants were only eligible if they had no signs or symptoms of any acute febrile disease in the previous eight weeks. We estimated a sample size for 1996 based on the population size at the time, and then in 2006, we repeated the sample size calculation with the updated population size; the samples for both studies was based on a prevalence of 0.5 with a maximum error of $5 \%$ and $95 \%$ bilateral confidence intervals $\left.\left[\mathrm{n}=\mathrm{NZ} \alpha^{2} /(\mathrm{N}-1)+\mathrm{Z} \alpha^{2} \mathrm{e}^{2}\right)\right]$, obtaining as a result a minimum sample of 352 for 1996 and 368 for 2006 . The sample was distributed to proportionally represent each age group.

For both serosurveys, blood samples consisted of $5 \mathrm{ml}$ of venous blood, drawn by conventional techniques from the forearm of the participants and transferred into Vacuteiner $R$ glass vials with serum separator; blood was preserved between 4 to 8 degrees Celsius to be subsequently centrifuged at 5,000 rpm for 10 minutes and finally transferred to the Institute of Epidemiological Reference (INDRE in Spanish). At the INDRE the serum was separated and tested for dengue IgG antibodies with Panbioßdengue IgG Indirect ELISA kits. Panbio $\mathbb{R}$ standardized unit cutoff points were used for negative (<9 UPB), indeterminate (9-11 UPB) and positive $(\geq 11 \mathrm{UPB}$ ) results, with specificity of 100 and sensitivity of 97.9 .

\subsection{Confirmed Denv cases between 1996 and 2006}

For the confirmed Denv cases reported between 1996 and 2006, we downloaded the morbidity annual records from the Board of Health, Department of Epidemiology web page, 
where DF and DHF cases are reported separately. Confirmed Denv cases between 1996 and 2006 were those with acute dengue clinical manifestations that were sampled and tested positive for IgM antibodies using Panbio $\AA$ Elisa that were finally registered in the Epidemiologic Surveillance System.

Table 1. Seropositivity to $\operatorname{IgG}$ indirect dengue Elisa in a random sample of Yucatan, Mexico

\begin{tabular}{|c|c|c|c|c|c|c|c|c|c|c|}
\hline \multirow{2}{*}{$\begin{array}{l}\text { Age } \\
\text { group }\end{array}$} & \multirow[t]{2}{*}{ Samples } & \multirow[t]{2}{*}{ Positive } & \multirow{2}{*}{$\begin{array}{l}\text { Proportion } \\
996\end{array}$} & \multicolumn{2}{|c|}{ CI 95\% } & Samples & Positive & Proportion & \multicolumn{2}{|c|}{ CI 95\% } \\
\hline & & & & & & & & 006 & & \\
\hline $0-4$ & 18 & 4 & 0.22 & 0.03 & 0.41 & 15 & 3 & 0.20 & 0.00 & 0.40 \\
\hline $5-14$ & 105 & 32 & 0.30 & 0.22 & 0.39 & 59 & 30 & 0.51 & 0.38 & 0.64 \\
\hline $15-24$ & 77 & 55 & 0.71 & 0.61 & 0.82 & 66 & 52 & 0.79 & 0.69 & 0.89 \\
\hline $25-44$ & 89 & 67 & 0.75 & 0.66 & 0.84 & 126 & 116 & 0.92 & 0.87 & 0.97 \\
\hline $45-64$ & 47 & 39 & 0.83 & 0.72 & 0.94 & 64 & 61 & 0.95 & 0.90 & 1.00 \\
\hline$\geq 65$ & 16 & 14 & 0.88 & 0.71 & 1.00 & 38 & 38 & 1.00 & 1.00 & 1.00 \\
\hline Total & 352 & 211 & 0.60 & 0.55 & 0.65 & 368 & 300 & 0.82 & 0.77 & 0.86 \\
\hline
\end{tabular}

\section{RESULTS}

The results of both dengue serosurveys in the state of Yucatan, Mexico in 1996 and 2006, show an overall seropositivity to Denv infection of 59.94\% in 1996 and $81.52 \%$ in 2006. Seropositivity increased by $21.58 \%$ in 2006 when compared to 1996 , that represent an annual average increase of $2 \%$ for the ten-year-period. Seropositive by age group and gender are detailed in Table 1.

While the 0-4-year-age group showed no increase in seropositivity between 1996 and 2006, an increment was observed in all other age groups, which means that seropositivity increased with age. It is important to mention that a slightly higher seroprevalence was found in males than females (see Figure 1).

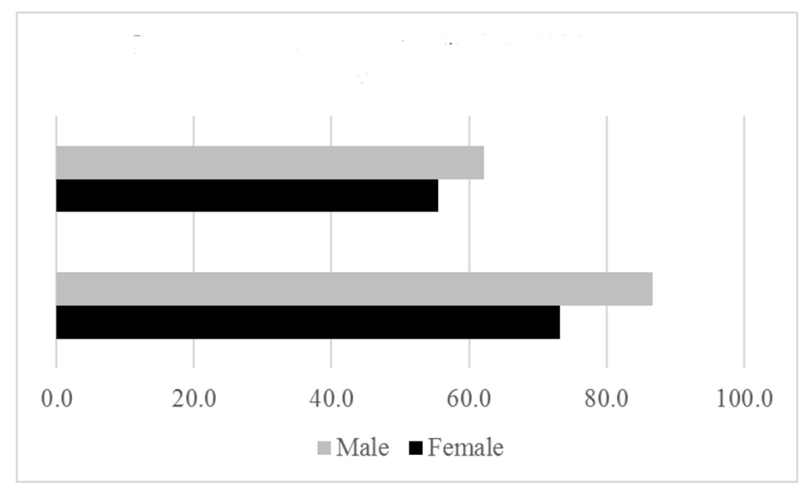

Figure 1. Overall seropositivity from 1996 and 2006 surveys by gender

A total of 6,443 DF cases in the state of Yucatan were reported to the Epidemiologic Surveillance System between 1996 and 2006 (see Table 2), additionally, 638 Dengue hem- orrhagic fever cases in Yucatan were also reported in that period, meaning that Dengue Hemorrhagic fever represented almost a $1 \%$ of all cases. It is important to mention that in 1997, an epidemic year, the proportion of DHF was lower (0.05) than in non-epidemic years (see Figure 2).

Table 2. Confirmed DF cases between 1996 and 2006 in Yucatan from epidemiologic surveillance

\begin{tabular}{llllllll}
\hline Year/Age & $\mathbf{0 - 4}$ & $\mathbf{5 - 1 4}$ & $\mathbf{1 5 - 2 4}$ & $\mathbf{2 5 - 4 4}$ & $\mathbf{4 5 - 6 4}$ & $\mathbf{2 6 5}$ & Total \\
\hline 1996 & 30 & 210 & 195 & 13 & 32 & 45 & 525 \\
1997 & 231 & 1,909 & 397 & 996 & 200 & 192 & 3,925 \\
1998 & 5 & 17 & 4 & 0 & 0 & 26 & 52 \\
1999 & 12 & 24 & 6 & 0 & 0 & 0 & 42 \\
2000 & 0 & 0 & 0 & 0 & 0 & 0 & 0 \\
2001 & 22 & 99 & 44 & 59 & 7 & 18 & 249 \\
2002 & 51 & 300 & 175 & 171 & 27 & 25 & 749 \\
2003 & 0 & 6 & 4 & 5 & 0 & 4 & 19 \\
2004 & 8 & 17 & 10 & 8 & 1 & 7 & 51 \\
2005 & 6 & 39 & 31 & 277 & 8 & 5 & 366 \\
2006 & 21 & 227 & 102 & 79 & 14 & 22 & 465 \\
Total & 386 & 2,848 & 968 & 1,608 & 289 & 344 & 6,443 \\
\hline
\end{tabular}

\section{Discussion}

Seropositivity to Denv in Yucatan increased dramatically in the ten-year-period elapsed between 1996 and 2006. Previous surveys in Mexico and other Latin American countries suggest that almost $65 \%$ of all infections are asymptomatic, and when adding the asymptomatic to the undiagnosed clinical cases, it could be estimated that up to $80 \%$ of DV infections remain unidentified by epidemiological surveillance system..$^{7,8]}$ 


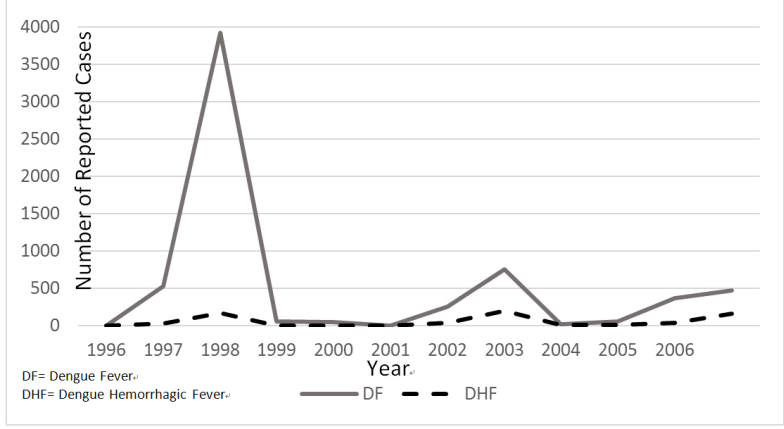

Figure 2. Degnue fever and dengue hemorrhagic fever cases in Yucatan reported between 1996 and 2006

In the present study we showed an increase in the population seropositivity between 1996 and 2006. This increase could not be otherwise estimated by analyzing DF and DHF reported cases alone, as seropositivity reflects not only symptomatic but also asymptomatic Denv infections. Anyway, by analyzing serosurveys results alone, retrospectively, we would not be able to identify the epidemic year of 1997, for that reason serosurveys and DF, DHF reported cases are information from different sources that complement each other, supporting the fact that passive and active surveillance need both to be implemented in order to have an ampler perspective of the Denv dynamics in the region.

Epidemiological surveillance of dengue, when developed by passive surveillance systems, involves that all suspected dengue cases must be reported and the laboratory confirmation of a proportion of those suspected cases, while active surveillance is useful to identify subclinical infections in the population, and it can include periodical serosurveys. ${ }^{[9,10]}$

Worldwide, national dengue epidemiological surveillance systems may vary, however, identification of acute cases is a common strategy; but in some countries, active surveillance strategies are developed during low transmission season, allowing the comparison of information and estimation of approximate numbers of incidence and evaluation of control strategies. In Mexico, serosurveys are not included in the dengue epidemiologic surveillance system. ${ }^{[10,11]}$

According to the World Health Organization, active surveillance can provide an accurate and pertinent warning for Dengue epidemics that can improve the preventive strategies, but it is important to use it effectively for planning an effective response, while passive surveillance systems alone are ineffective for detecting an epidemic much before peak transmission. If we put the data we analyzed in the present study as an antecedent of what happened in the years following the last serosurvey, we can imply that if serosurveys were developed periodically, and if they included serotype specific immunity analyses (PRNT) along with vector surveillance, the Mexican epidemiologic bureau could have been able to predict early the outbreaks and it could have been able to plan specific strategies to reduce the burden of dengue in the region. ${ }^{[12,13]}$

Twenty and ten years ago, Yucatan had not reached the actual levels of urban population density, which has implications due to the fact that the presence of the vector and the viral transmission are intensified by the rapid urban growth. Nowadays $84 \%$ of the state population live in urban areas, that is why updated serosurveys could be useful to determine the contemporary population seropositivity for Denv infections in the region. It is not late for the Mexican health agencies to improve the dengue surveillance system, including the implementation of periodic serosurveys in endemic regions as a part of a complete, holistic approach, in order to not only predict the occurrence of an outbreak, but also to evaluate the impact of DF and DHF programs. ${ }^{[14-16]}$

\section{Conclusions}

Data drawn from the 1996 and 2006 serosurveys showed an increase of seropositivity to Denv infections, which can partially be explained by the 1997 outbreak in the Yucatan. While seroprevalence studies were useful to identify the proportion of seropositive population, the case reports from the epidemiologic surveillance system were useful to identify the epidemic year, meaning that both sources of information are complementary to better understand the Denv dynamics during the ten-year-period elapsed between 1996 and 2006.

\section{Limitation}

There are several limitations that need to be considered in the present study. The main limitation derives from the time when the information for this study was collected, as it has passed twenty years since the generation of the first serosurvey and ten years since the second serosurvey was developed. During that time, the population and environmental aspects related to dengue as a vector borne disease, have changed. Our data need to be interpreted with caution considering the time elapsed.

Another aspect to consider is that the epidemiologic surveillance systems have changed with time, and some imprecisions could derive from these changes in the report of DF and DHF cases in the state of Yucatan. Even when diagnostic capacity and methods for diagnosis have not changed, the case reports 20 years ago were mostly made in paper formats and faxed to the epidemiologic surveillance departments in the city of Mexico to be entered in the national database. With the widespread access to internet and the National Epidemio- 
logic Surveillance platform, case reports are now uploaded in real time.

Finally, limitations associated with the laboratory diagnosis need also to be kept in mind, as only Denv antibodies were considered to establish seropositivity among individuals and also to identify DF, DHF cases. Sensitivity and specificity of IgM and IgG antibodies should be considered along with the possibility of crossed reactivity to other flaviviruses. Never- theless, no other flaviviruses circulated in the region for the previous seven decades (yellow fever occurred in Yucatan in the early 1920s). Clinical manifestations and migration antecedents were asked to the participants and correlated with the laboratory results in all cases.

\section{CONFlicts of InTEREST Disclosure}

Authors declare that they have no competing interests.

\section{REFERENCES}

[1] Loroño Pino MA, Farfán Ale JA, Rosado Paredes EdP, et al. Epidemic dengue 4 in the Yucatan, Mexico, 1984. Revista do Instituto de Medicina Tropical de Sao Paulo. 1993; 35(5): 449-55. http://dx.doi.org/10.1590/S0036-46651993000500011

[2] Loroño-Pino M, Farfan-Ale J, del-pilar-Rosado-Paredes E, et al. Prevalencia de anticuerpos contra virus dengue en Yucatan, México, 1985. Revista Latinoamericana de Microbiología. 1989; 31(4): 259-62.

[3] CDC. (Centers for Disease Control and Prevention) Dengue-Mexico. Morbidity and Mortality Weekly Report. 1979; 28: 402-404.

[4] Toledo-Romaní ME, Baly-Gil A, Ceballos-Ursula E, et al. Participación comunitaria en la prevención del dengue: un abordaje desde la perspectiva de los diferentes actores sociales. Salud Pública de México. 2006; 48(1): 39-44. PMid:16555533 http://dx . doi .org /10.1590/S0036-36342006000100007

[5] Secretaría de Salud. Dirección de Vigilancia Epidemiológica de Enfermedades Transmisibles. Panorama Epidemiológico de Dengue 2015. Available from: http://www.epidemiologia.salud.gob. $\mathrm{mx} /$ dgae/panodengue/intd_dengue_2015.html. 2016

[6] Méndez-Galván J, Fernández-Cerna F. Workshop on recent advances in community-based Aedes aegypti control: Honduras and México. Mérida, Yucatán, México 1996. (Memorias y material videograbado): Secretaría de Salud de México y Ministerio de Salud Pública de Honduras E. Supported by Rockefeller Foundation.

[7] Halstead S. Pathogenesis of Dengue: Dawn of a New Era. F1000 Research. 2015; 4(1353). PMid: 10.12688

[8] Rothman AL. Dengue: defining protective versus pathologic immunity. The Journal of Clinical Investigation. 2004; 113(7): 946-51. PMid:15057297 http://dx.doi.org/10.1172/JCI21512
[9] Kourí G, Guzmán MG, Valdés L, et al. Reemergence of dengue in Cuba: a 1997 epidemic in Santiago de Cuba. Emerging Infectious Diseases. 1998; 4(1): 89. PMid:9454563 http://dx.doi .org/10. 3201/eid0401.980111

[10] Lai S, Huang Z, Zhou H, et al. The changing epidemiology of dengue in China, 1990-2014: a descriptive analysis of 25 years of nationwide surveillance data. BMC Medicine. 2015; 13(1): 100. PMid:25925417 http://dx.doi.org/10.1186/s12916-015-0336-1

[11] Beatty ME, Stone A, Fitzsimons DW, et al. Best practices in dengue surveillance: a report from the Asia-Pacific and Americas Dengue Prevention Boards. PLoS Negl Trop Dis. 2010; 4(11): e890. PMid:21103381 http://dx.doi.org/10.1371/journal.pntd. 0000890

[12] Gubler DJ. Aedes aegypti and Aedes aegypti-borne disease control in the 1990s: top down or bottom up. The American Journal of Tropical Medicine and Hygiene. 1989; 40(6): 571-8. PMid:2472746

[13] Gubler DJ. How effectively is epidemiological surveillance used for dengue programme planning and epidemic response? Dengue Bulletin. 2002; 26: 96-106.

[14] Iturrino-Monge R, Avila-Agüero ML, Avila-Agüero CR, et al. Seroprevalence of dengue virus antibodies in asymptomatic Costa Rican children, 2002-2003: a pilot study. Revista Panamericana de Salud Pública. 2006; 20(1): 39-43. PMid:17018223 http://dx.doi .org /10.1590/S1020-49892006000700005

[15] Telle O, Vaguet A, Yadav NK, et al. The Spread of Dengue in an Endemic Urban Milieu-The Case of Delhi, India. PLoS ONE. 2016; 11(1): e0146539. http://dx.doi.org/10.1371/journal.pon e. 0146539

[16] Gubler DJ. Dengue, urbanization and globalization: the unholy trinity of the 21 st century. Tropical Medicine and Health. 2011; 39(4SUPPLEMENT): S3-S11. PMid:22500131 http://dx.doi.org/10. 2149/tmh.2011-S05 